\title{
Highly efficient integrated rectifier and voltage boosting circuits for energy harvesting applications
}

\author{
D. Maurath, C. Peters, T. Hehn, M. Ortmanns, and Y. Manoli \\ Chair of Microelectronics, Department of Microsystems Engineering (IMTEK), University of Freiburg, \\ Georges-Koehler-Allee 102, 79110 Freiburg, Germany
}

\begin{abstract}
This paper presents novel circuit concepts for integrated rectifiers and voltage converting interfaces for energy harvesting micro-generators. In the context of energy harvesting, usually only small voltages are supplied by vibration-driven generators. Therefore, rectification with minimum voltage losses and low reverse currents is an important issue. This is realized by novel integrated rectifiers which were fabricated and are presented in this article. Additionally, there is a crucial need for dynamic load adaptation as well as voltage up-conversion. A circuit concept is presented, which is able to obtain both requirements. This generator interface adapts its input impedance for an optimal energy transfer efficiency. Furthermore, this generator interface provides implicit voltage up-conversion, whereas the generator output energy is stored on a buffer, which is connected to the output of the voltage converting interface. As simulations express, this fully integrated converter is able to boost ac-voltages greater than $|0.35 \mathrm{~V}|$ to an output dcvoltage of $2.0 \mathrm{~V}-2.5 \mathrm{~V}$. Thereby, high harvesting efficiencies above $80 \%$ are possible within the entire operational range.
\end{abstract}

\section{Introduction}

Especially electronic devices participate the development towards miniaturization and high performance with least power consumption only. Nowadays, very low power applications emerge increasingly in the field of stand-alone and wireless acting sensor/actuator-systems. A further rise of the applicability of such systems as fully embedded nodes suffers from the inconvenience with batteries. These electrochemical energy storages have limited lifespan, are very temperature sensitive and need to be replaced repetitively. In case of hardly

Correspondence to: D. Maurath

(maurath@imtek.de) accessible locations, there is little chance for having battery power supply based cost-efficient wireless embedded systems. Thus, the idea of power supplies, which are based on energy harvesting principles, is a helpful contribution in order to realize cost-efficient fully embedded sensor/actuatorsystems.

A very space-efficient and robust kind of energy harvesting is possible with vibration-driven inductive microgenerators. However, such micro-generators supply only small open-circuit output voltage in the range of $2-3 \mathrm{~V}_{p p}$. Beside this, a relatively high internal resistive impedance causes only a small load optimum, were the maximum possible output power is obtained. These circumstances enforce developments of novel kinds of rectifiers, since common fullwave rectifiers cause relatively high voltage losses.

In order to circumvent these crucial limitations, the second chapter shows enhanced rectifier circuitry with active diodes, which were designed, implemented and fabricated. These active diodes consist of an active circuit instead of passive devices only. Additionally, due to this low voltages and high internal resistance an adaptive generator-interface is required. This interface gives the opportunity of most appropriate loading of the generator. Since only a simple capacitive energy buffer connected to the output of the rectifier can not provide adequate load matching. Due to the realization of this interface with switched-capacitors, the functionality of the rectifier is improved with respect to minimum input voltages. Conclusively, the forth chapter presents simulated as well as measured results. So, the active diodes of the proposed rectifier without the generator interface can operate above $1.75 \mathrm{~V}$ generator output voltage amplitude. Together with the interface, generator output amplitudes of less than $1 \mathrm{~V}$ are sufficient for operation. Whereas the rectification, matching and conversion efficiencies can be kept above $80 \%$ over the entire operating range. 


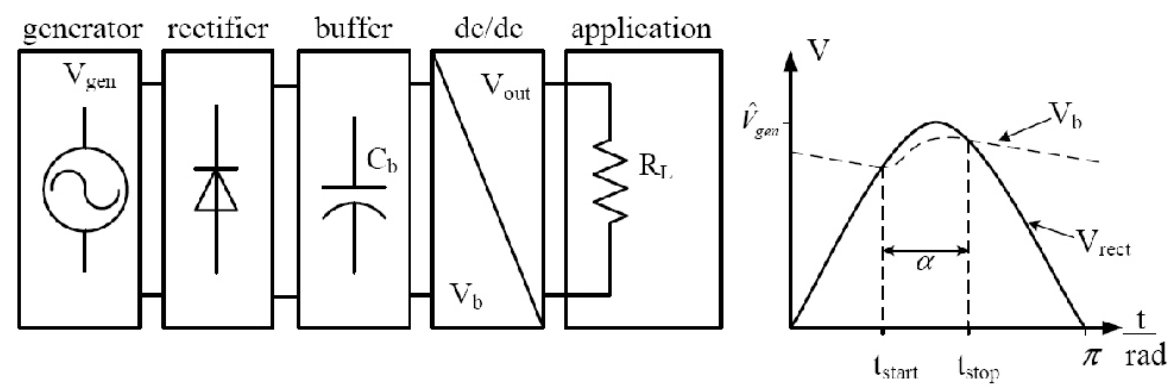

Fig. 1. Common single stage power conversion and generator interfacing approach (a). The generator can only supply power within conduction degree $\alpha(\mathbf{b})$.

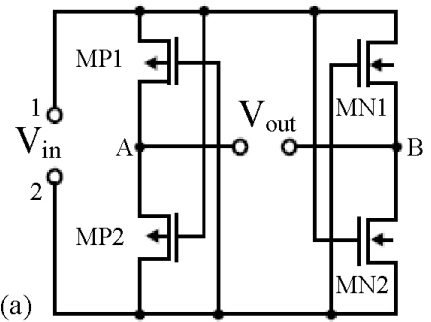

(b) Diode-connected PMOS

(c)

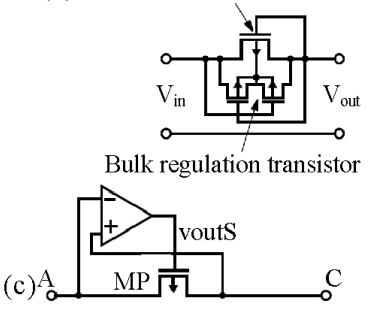

Fig. 2. Schematic of a negative voltage converter (a), the bulk regulation configuration (b), and the active diode principle (c).

\section{Conventional harvesting interfaces}

Common generator interfacing configurations are comparable to the configuration shown in Fig. 1a. Together with this simple structure also conventional circuit principles are usually applied. In case of batteries as power sources, this approach can be very useful and efficient. However, in case of energy harvesting with micro-generators this approach may exhibit crucial drawbacks.

\subsection{Conventional rectifier circuits}

Conventional full-wave rectifiers suffer a relatively high and unappreciated voltages loss of about $1.4 \mathrm{~V}$. In case of Schottky diodes, that voltage loss could be reduced to around $0.6 \mathrm{~V}$, however, those diodes have intrinsically higher reverse current, which is also not acceptable. Concerning integrated rectifiers, the diodes are realized with MOSFETs, because pn junctions are often not specified in forward direction in CMOS processes. The bulks of PMOS transistors must always be connected to the highest potential and of NMOS to the lowest to avoid leakage currents and latch-up. A dynamic bulk regulation (Fig. 2b), consisting of two transistors, avoids this and must be added to every transistor. Due to this, the number of transistors and leakage increases.

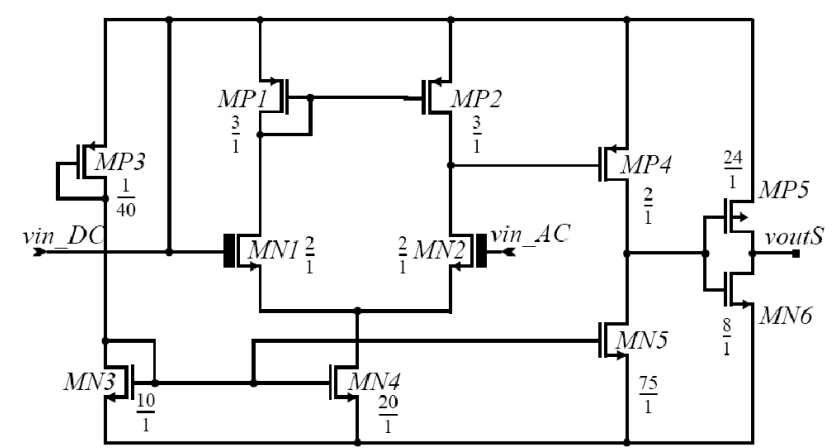

Fig. 3. Schematic of the comparator circuit with differential stage, common-source output amplifier and inverter stage.

Similarly, conventional full wave (F_W) rectifiers use four MOSFETs connected as diodes. Every half wave is used, but the voltage drop is twice $V_{t}$ and strongly reduces the available output voltage. A smart improvement is presented in (Ghovanloo and Najafi, 2004), which replaces two diode-connected transistors with two cross-coupled transistors. Thus, only one $V_{t}$ voltage drop is lost.

\subsection{Common conversion concepts}

The common approach, as depicted in Fig. 1, is reasonable in case of some energy storage elements, like a battery, as a power source. However, if micro-generators are considered, power can be gathered only when the generator is exited. That means, in order to be able to supply an application independent of the generator excitation, two concerns are necessary. First, if the generator is excited most possible energy has to be harvested. Second, most of that maximum possible energy need to be stored at some energy storage element, like a buffer capacity. Additionally, in order to harvest continuously the maximum possible energy, permanent optimal generator loading for maximum generator output power as well as efficient rectification, and voltage conversion are required (Shengwen et al., 2005). 


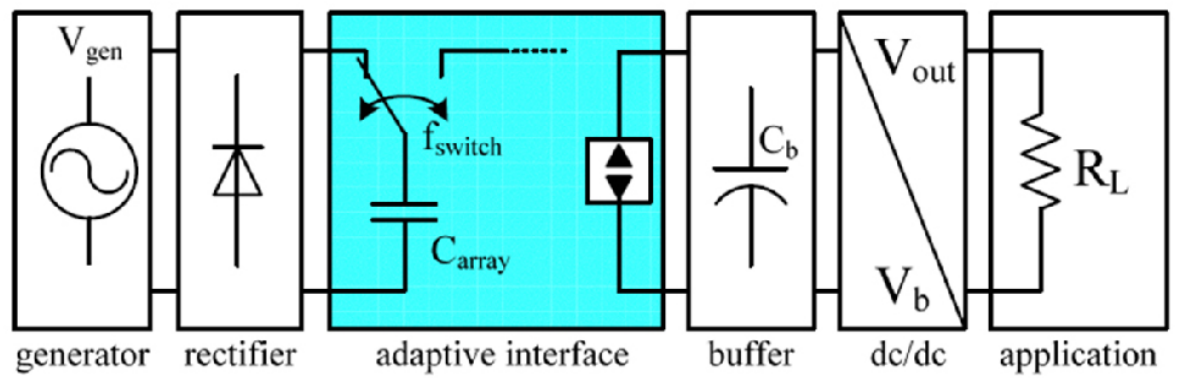

Fig. 4. Building blocks of the two stage approach, which is includes an additional load-adaptive generator interface into the power conversion chain.

\section{Proposed harvesting circuitry}

The generator interface was especially designed to incorporate with inductive micro-generators (Spreemann, 2006; Kulkarni et al., 2007). The internal source resistance of these inductive generators is between $R_{i}=1-5 k \Omega$. The generated open-circuit output voltage has $2.8 V_{p p}$ at most and frequencies are not higher then $f_{\text {gen }}=500 \mathrm{~Hz}$.

\subsection{Novel active rectifier}

The main goals of this novel rectifier are the reduction of the voltage drop over the MOSFETs and achievement of a high efficiency. This rectifier can be separated into two stages: (i) the negative voltage converter and (ii) the active diode part.

\subsubsection{First stage: negative voltage converter $(\mathrm{CON})$}

The first stage of the proposed rectifier circuit is used to convert the negative half waves of the input sinusoidal wave into positive ones. This conversion is done with only four standard CMOS transistors (see Fig. 2a). No special technology, e.g. isolated transistors, is needed to realize this circuit. The left side is realized with PMOS transistors and always delivers the highest potential at $V_{i n}$ to node A. The right side consists of NMOS transistors and the low voltage potential is at point B. No additional dynamic bulk regulation transistors are necessary, because this is inherently given in this circuit. Thus, the bulk of the PMOS transistors can be directly connected to point $\mathrm{A}$ and the NMOS to point $\mathrm{B}$. This reduces the number of required transistors.

The ability to convert nearly the entire voltage applied at the input to the output is the main advantage of this circuit. The voltage drop with this rectifier is less than $10 \mathrm{mV}$ (Peters et al., 2007) and the negative half wave is now converted into a positive one. But this circuit can not be used to charge a storage capacitor, because the current direction is not controlled and current back flow occurs. Due to this a second stage is necessary to realize a useful rectifier.

\subsubsection{Second stage: active diode current barrier (AD)}

The main function of the second rectifier stage is to control the current direction. This could be in the simplest case a conventional diode-connected MOSFET. Using this, a full wave rectifier with only one voltage drop $V_{t}$ over the transistor results (CON_D). This circuit is comparable to the full wave rectifier with switches (FW_S), but it is less prone to latch-ups and uses only 7 compared to 12 transistors.

In order to achieve very high output voltages and high efficiencies the diode should be replaced by another element, the active diode. This active diode works nearly as an ideal diode, with current flowing in only one direction and nearly no voltage drop. The main difference to an ideal diode is the current consumption of the device. A complex realization of an active diode is presented in Lehmann and Moghe (2005). A control circuit is used to determine the conducting and non conducting time of a MOSFET used as a switch. Lam et al. (2005) needs two active diodes to realize a full wave rectifier. The active diode presented in this paper is simpler and based on a fast and low power comparator circuit (see Fig. 2c). MP is the switch which is controlled by the comparator. If the voltage at the anode is higher compared to the cathode the output of the comparator is $0 \mathrm{~V}$ and MP is on. If the cathode is higher the output is high and MP is off. The supply voltage of the comparator is taken from the storage capacitor. Using a PMOS transistor as switch no additional start-up circuit is necessary for this active diode.

The most important part of the active diode is the comparator. A fast comparator with a low power consumption is needed. If the comparator is too slow not all available energy can be transferred to the capacitor and additionally current back flow occurs after the input voltage has passed the maximum value. The implemented comparator (see Fig. 3) is simply based on a differential stage, a common-source output amplifier and an inverter. The input $v_{\mathrm{in}, \mathrm{DC}}$ is connected to the cathode (C) (Fig. 2c) and is also the power supply of the comparator. $v_{i n, A C}$ is connected to the alternating sinusoidal input wave (anode (A)). The overall current consumption is less than $1 \mu \mathrm{A}$ for an input voltage of $\pm 2.75 \mathrm{~V}$. The operation 


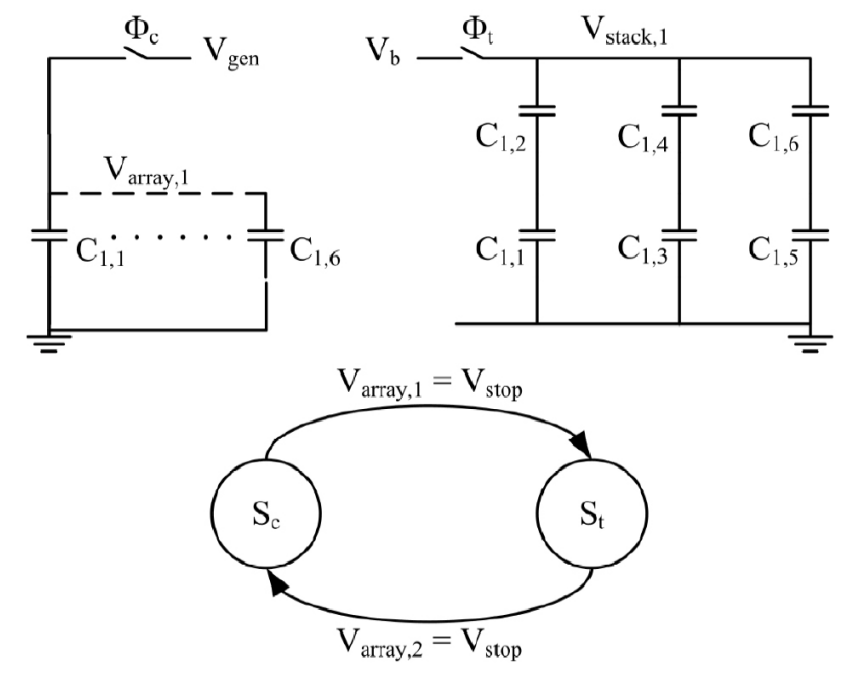

Fig. 5. Schematic and state diagram of a conversion cycle cycle.

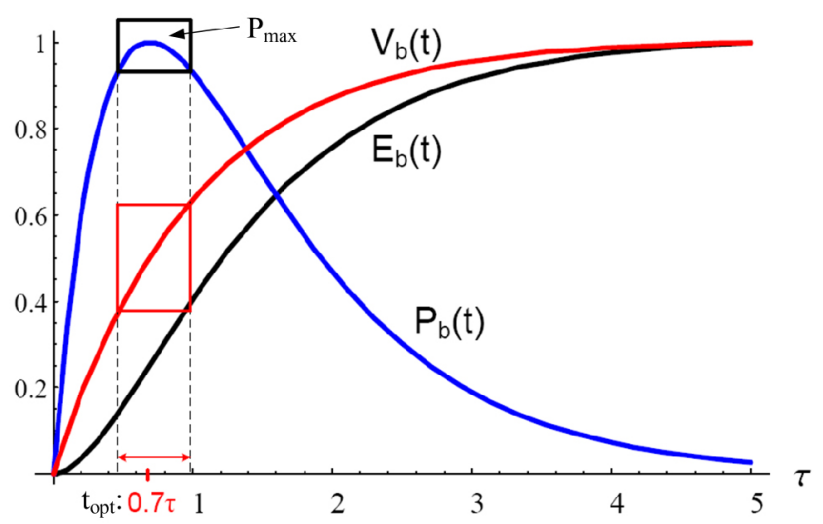

Fig. 6. This plot shows the transient power transfer while an empty capacitor is charged.

voltage range is between $1.6 \mathrm{~V}$ and $3.75 \mathrm{~V}$. Combination of the negative voltage converter (CON) (Fig. 2a) with the active diode (Fig. 2c) overcomes the mentioned drawback.

\subsection{Capacitive generator interface and converter}

In order to harvest continuously the highest possible energy, the power electronic is again separated in two stages (see Fig. 4). The first stage is a generator interface and has to be optimized for adaptive impedance matching so as to load the generator as ideal as possible. In addition to that, this first stage also has to transfer the harvested energy most efficiently into a buffer. Thereby, the output voltage of the first stage is neither fixed nor regulated by the converter. For efficient energy transfer, the output voltage is rather adapted to the buffer voltage $V_{b}(t)$. With appropriate dimensioning of the components, the buffer voltage will vary only little and

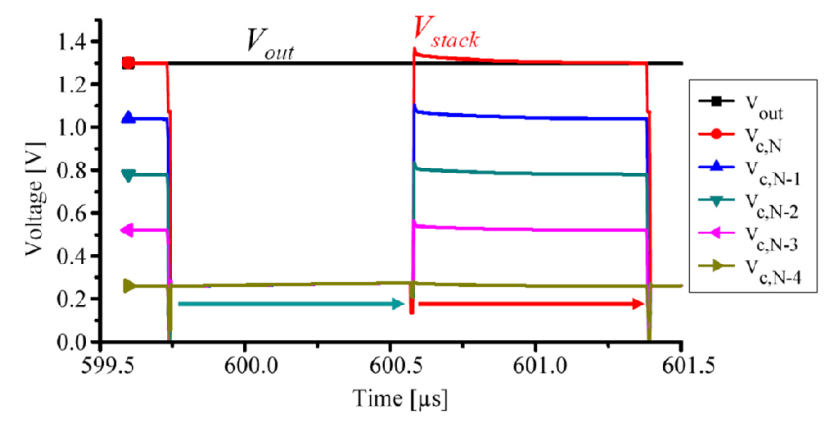

Fig. 7. This graph shows one conversion cycle, whereas $V_{\text {array }}$ is the highest voltage within the array.

slowly compared to the generator ac voltage. Thus, the second stage is realized as a dc/dc converter.

The here presented generator-interface works basically as a voltage converter with adaptive input impedance adaptation, efficient energy transfer and implicit voltage upconversion. By applying power saving circuit techniques, the power consumption of the control circuitry and switch driving should be less then $P_{\text {total }} \leq 50 \mu \mathrm{W}$.

\subsubsection{Adaptive conversion principle}

The proposed adaptive switching converter is based on two capacitor arrays, which toggle complementary-phased with frequency $f_{\text {conv }}$ between a charging and a transfer state, $S_{c}$ and $S_{t}$, respectively (see Fig. 5). Whereas, a conversioncycle is the single succession of $S_{c}$ and $S_{t}$. In the considered converter both arrays consist of $k_{\max }=6$ capacitors, whereas $k_{\max }$ directly corresponds to the maximum required voltage conversion ratio. However, the number of array capacitors $n_{i}$, which are applied within a single conversion-cycle, is dynamically adapted according to the current required conversion ratio $k_{i}=V_{b} / V_{\text {gen }}(t)$. Hence, while one array is in charging state $S_{c}, n_{i}$ array capacitors out of the $k_{\max }$ available array capacitors are connected to the generator in parallel. At this state, the capacitors are charged until the capacitor voltages $V_{\text {array }}$ reach a certain voltage limit $V_{\text {stop }}$, as shown in Fig. 7. Afterwards, the array transitions to transfer state $S_{t}$. Therefore, the array capacitors have to be re-configured so as to obtain a stacked voltage $V_{\text {stack }}$, which is higher than the buffer voltage $V_{b}$ of an external buffer. In order to obtain the dynamic adaptation of the optimal conversion ratio $k_{i}$, the converter works in an oversampling manner. Thus, if $f_{\text {gen }} \ll f_{\text {conv }}$. is given, the conversion ratio can be adapted quite continuously within a generator voltage period $1 / f_{\text {gen }}$.

\subsubsection{Adaptive impedance matching}

High internal generator resistance $R_{i}$ requires a particular converter design since an exceeding current load would cause a dramatic voltage breakdown, which would cause reduced 


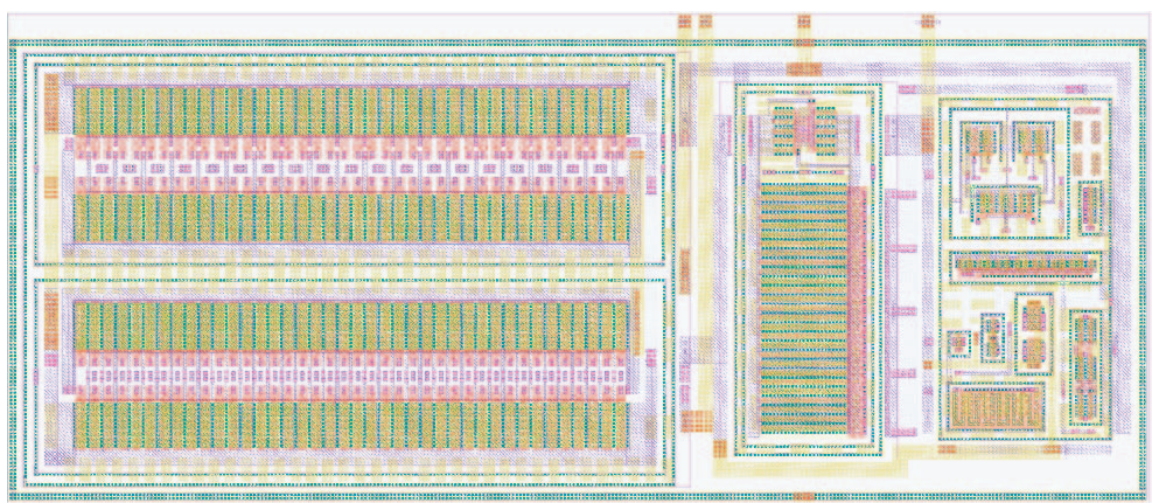

Fig. 8. The layout of the active diode enhanced rectifier, whereas the dimensions are $75 \times 40 \mu \mathrm{m}$.

generator output power. Due to the low output currents of energy harvesting generators $(50-500 \mu \mathrm{A})$ capacitive voltage conversion is preferred rather than inductive conversion. However, charging a capacitor allows generally only at $t_{\mathrm{opt}} \approx \ln (2)$ maximum power transfer from the generator into the capacitor (see Fig. 6). The generator-interface matching situation at $t_{\mathrm{opt}}$ is equivalent to the impedance matching situation where $P_{\text {gen }}\left(R_{i}=R_{\text {load }}\right)=P_{\max }$ is achieved. Therefore, for optimal operation the capacitors charging level should stay around a optimal voltage of $V_{\mathrm{ch}, \text { opt }} \approx V_{\text {gen }}(t)$, which corresponds to $t_{\text {opt }}$. This means, within $S_{c}$ the generator charges the capacitors up to $V_{\text {stop }}=V_{\text {ch,opt }}+\Delta V_{c h}$. Then the capacitors are toggled to $S_{t}$ and discharged onto an output buffer $C_{b}$ until the capacitor voltage reaches, $\Delta V_{\text {ch }}$ and $\Delta V_{\text {disch. }}$. The consequence of keeping the caps only in a small charging range of $0.5 V_{\mathrm{gen}}(t) \pm \Delta V$ requires an increased number of capacitors. Thus, twice as many capacitors are implemented to boost the generator voltage up to a certain voltage.

\subsubsection{Voltage conversion and array configuration}

Due to the fact, that charge transfer from one capacitor to another one causes intrinsic losses due to series resistances (Gobbi et al., 2006). But by minimizing the initial voltage gap between $V_{\text {stack }}$ and $V_{b}$ the charge transfer losses are minimized. Hence, the optimal conversion ratio $k_{i}$ is obtained by

$k_{i}=\frac{V_{b}}{V_{\text {ch,opt }}+\Delta V_{\text {ch }}}=\frac{V_{b}}{V_{\text {stop }}}$

The strategy for stacking is that a maximum quantity $n_{i} \rightarrow k_{\max }$ of capacitors is used, whereas within each stackstage $n_{i} / k_{i}$ capacitors are connected in parallel (see Fig. 5). Thus, the maximum capacitance for a certain conversion ratio is used, which reduces the conversion frequency $f_{\text {conv }}$.

\section{Results}

Generally, by application of the proposed energy harvesting circuit concepts more energy can be extracted out of the same micro-generator. The power and voltage losses are reduced and the load matching condition is improved.

\subsection{Rectification efficiencies}

The main aspects of rectifiers are the maximum output voltage which can be reached and the efficiency $\eta_{\text {rect }}$, which is simply the quotient of the output and the input energy over one period. The maximum achievable output voltage is especially important for energy harvesting with inductive microgenerators.

The circuits have been simulated and implemented with Cadence using a $0.35 \mu \mathrm{m}$ HV CMOS C35 process from austriamicrosystems (see Fig. 8). The voltage and efficiency values strongly depend on the used frequency, storage capacitor and ohmic load. Figure 9 shows the transient behavior of the discussed rectifiers. For these simulations the frequency is $125 \mathrm{kHz}$, a capacitance of $500 \mathrm{pF}$ and a load of $20 \mathrm{k} \Omega$ are used. For useful applications a larger capacitance value should be used to reduce the voltage ripple. But this value is more suitable for showing the excellent performance of the proposed rectifiers.

\subsubsection{Rectifier output voltage comparison}

Figure 9 shows the input and the output wave form of the discussed rectifiers. In this case the open circuit input voltage was $\pm 2.75 \mathrm{~V}$. The negative voltage converter in combination with a PMOS diode (CON_D) has nearly the same voltage characteristic as the FW_S. Both rectifiers with the active diode (HW_AD and CON_AD) reach nearly the input voltage. The minimum voltage of the CON_AD rectifier is also very high, because every half wave is used and the capacitor is charged up twice at each period. 


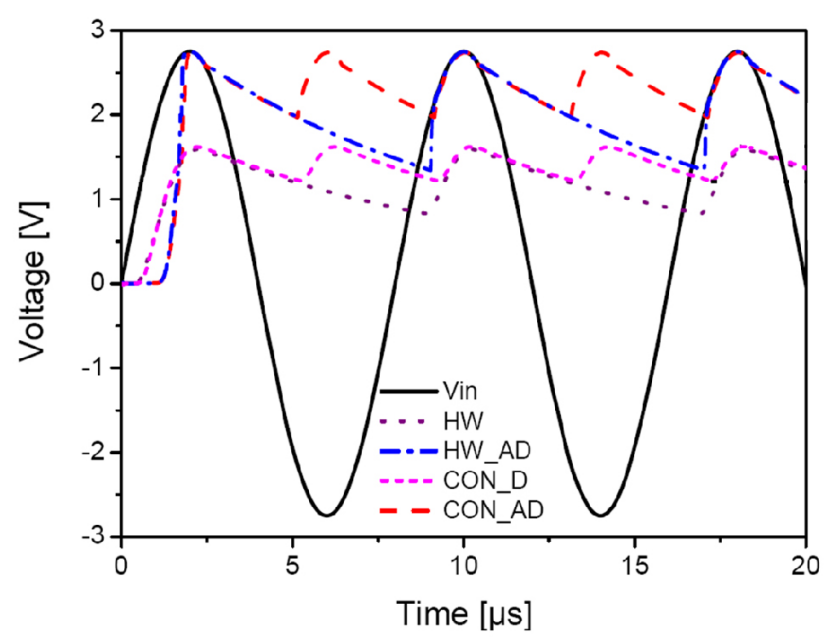

Fig. 9. Simulation results of the output signal of the compared rectifiers. The output voltage range of CON_D is in average less than the output voltage range of CON_AD.

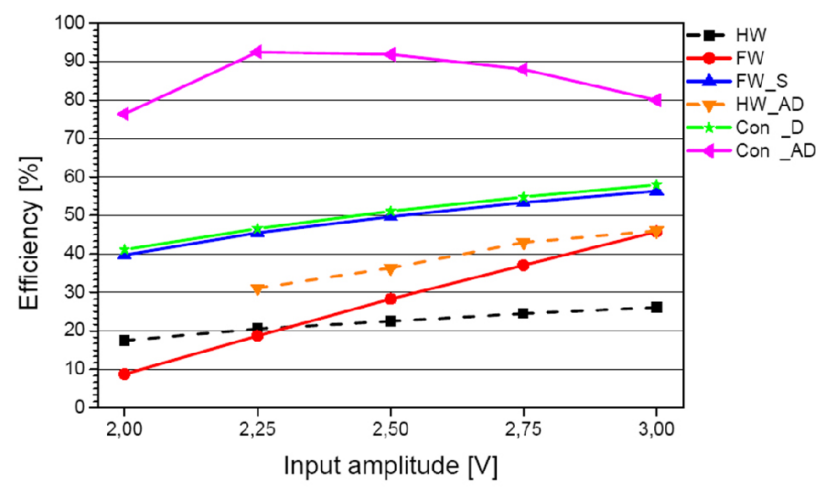

Fig. 10. Efficiencies of the compared rectifiers plotted versus the input voltage.

\subsubsection{Enhanced rectification efficiency}

Beside the attainable output voltage the efficiency is also of importance. The efficiency of the different rectifiers versus the input amplitude is shown in Fig. 10. The use of an active diode in a half wave rectifier shows a strong increase of the efficiency (HW vs. HW_AD). The highest efficiencies could be reached using the negative voltage converter in combination with the active diode (CON_AD). The efficiency in this example is always larger than $75 \%$.

\subsection{Interface matching and conversion results}

Within all simulations and calculations, the generator was included as a sine wave source with an ohmic serial resistance. Because energy harvesting applications are considered, there

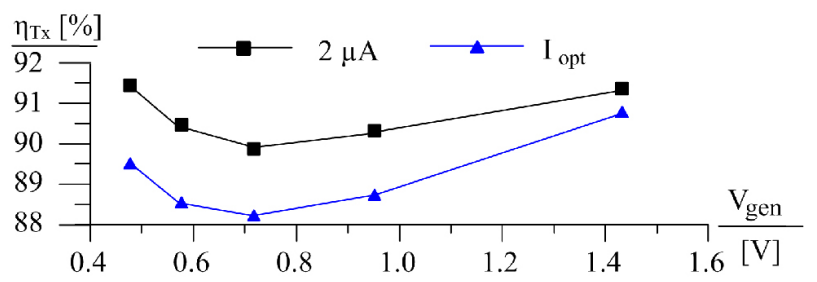

(a)

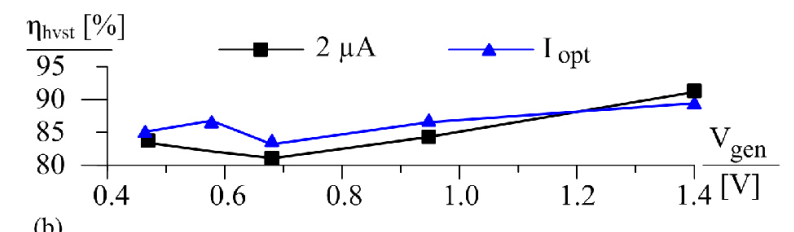

(b)

Fig. 11. The simulated efficiencies in graph (a) show the ratio of harvested power compared to maximum possible power, and (b) shows the overall conversion efficiency.

are two important efficiencies:

$$
\begin{aligned}
& \eta_{T x}=\frac{1}{\left(t_{2}-t_{1}\right)} \frac{\int_{t_{1}}^{t_{2}} V_{b} I_{c h} \mathrm{~d} t}{\frac{\hat{V}_{\text {gen }}}{4 R_{i}}} \\
& \eta_{\text {hvst }}=\eta_{T x} \times \eta_{\text {conv }}=\eta_{T x} \frac{\int_{t_{1}}^{t_{2}} V_{\text {load }} I_{\text {load }} \mathrm{d} t}{\int_{t_{1}}^{t_{2}} V_{\text {gen }} I_{\text {gen }} \mathrm{d} t}
\end{aligned}
$$

Where Eq. (2) defines the input efficiency $\eta_{T x}$, which compares the theoretical maximum generator power to the actual converter input power. The higher $\eta_{T X}$ is, the better achieved is impedance matching. In addition to this, the efficiency $\eta_{\text {hvst }}$ in Eq. (3) gives a measure of the complete harvesting efficiency. This efficiency is mainly affected by internal converter losses and capacitor charge transfer losses. As Fig. 11a illustrates, the interface input power almost equals the maximum possible output power.

Both graphs prove that this novel interface approach and conversion method operates as required. Generally, in order to characterize the efficiency by interfacing a generator, not only $\eta_{\text {conv }}$ can be considered, but also $\eta_{T x}$ is of importance. Therefore, Fig. 11b shows the overall harvesting efficiency $\eta_{\text {hvst }}$ over the generator voltage range.

\section{Conclusions}

A novel rectifier concept consisting of a voltage converter and an active diode was presented. No additional bulk regulation transistors are needed and nearly the entire generator voltage appears at the rectifier output. In combination with a MOSFET diode the efficiency is about $50 \%$. In combination with an active diode rectifier efficiencies over $90 \%$ can 
be reached. The maximum output voltage is only a few $\mathrm{mV}$ below the input voltage.

It was also shown, that high efficient power extraction and voltage up-conversion is feasible in energy harvesting environments. Especially the realization of adaptive impedance matching and almost continuous maximum generator power enhances the usage of micro-generators further. These advantages give the opportunity of using smaller generators to supply autonomous applications. One drawback might be the relatively large quantity of capacitances, which need to be integrated. Thus, a lot of chip area is necessary and parasitic effects could decrease the overall efficiency.

Acknowledgements. This work is supported by the German Research Foundation (Deutsche Forschungsgemeinschaft - DFG) under Grant Number GR1322 and MA 2193/7-1.

\section{References}

Ghovanloo, M. and Najafi, K.: Fully integrated wideband highcurrent rectifier for inductively powered devices, IEEE J. Solid State Circuits, 39, 1976-1984, 2004.

Gobbi, L., Cabrini, A., and Torelli, G.: Impact of Parasitic Elements on CMOS Charge Pumps: a Numerical Analysis, IEEE International Symposium on Circuits and Systems (ISCAS), May 2006.
Kulkarni, S., Koukharenko, E., Tudor, J., Beeby, S., O’Donnell, T., and Roy, S.: Fabrication and Test of Integrated Micro-Scale Vibration Based Electromagnetic Generator, International SolidState Sensors Actuators and Microsystems Conference, 2007.

Lam, Y.-H., Ki, W.-H., and Tsui, C.-Y.: Integrated Low-Loss CMOS Active Rectifier for Wirelessly Powered Devices, IEEE Trans. Circuits and Systems, 53(12), 1378-1382, 2006.

Lehmann, T. and Moghe, Y.: On-chip active power rectifiers for biomedical applications, IEEE International Symposium on Circuits and Systems, 1, 732-735, 2005.

Peters, C., Kessling, O., Henrici, F., Ortmanns, M., and Manoli, Y.: CMOS Integrated Highly Efficient Full Wave Rectifier, IEEE IEEE International Symposium on Circuits and Systems (ISCAS), 2415-2418, New Orleans, 2007.

Shengwen, X., Ngo, K. D. T., Nishida, T., Gyo-Bum, Ch., and Sharma, A.: Converter and controller for micro-power energy harvesting, 20th Annual Applied Power Electronics Conference and Exposition, 1, 226-230, March 2005.

Spreemann, D., Folkmer, B., Maurath, D., and Manoli, Y.: Tuneable transducer for low frequency vibrational energy harvesting, EuroSensors 2006, September 2006. 